\title{
Association of intraindividual tacrolimus variability with de novo donor-specific HLA antibody development and allograft rejection in pediatric kidney transplant recipients with low immunological risk
}

\author{
Maral Baghai Arassi ${ }^{1,2}$ D $\cdot$ Laura Gauche $^{1} \cdot$ Jeremy Schmidt $^{1} \cdot$ Britta Höcker $^{1}$ (D) $\cdot$ Susanne Rieger $^{1} \cdot$ Caner Süsal $^{3}$ (D) . \\ Burkhard Tönshoff ${ }^{1}$ (D) Alexander Fichtner ${ }^{1}$ (iD
}

Received: 30 June 2021 / Revised: 24 November 2021 / Accepted: 13 December 2021 / Published online: 15 February 2022

(c) The Author(s) 2022

\begin{abstract}
Background Tacrolimus (Tac) intraindividual variability (TacIPV) in pediatric kidney transplant patients is only poorly understood. We investigated the impact of TacIPV on de novo donor-specific HLA antibodies ( $d n$ DSA) development and allograft rejection in Caucasian pediatric recipients of a living or deceased donor kidney with low immunological risk.

Methods This was a single-center retrospective study including 48 pediatric kidney transplant recipients. TacIPV was calculated based on coefficient of variation (CV\%) 6-12 months posttransplant. TacIPV cutoff was set at the median (25\%). Outcome parameters were $d n \mathrm{DSA}$ development and rejection episodes.

Results In total, 566 Tac levels were measured with median 11.0 (6.0-17.0) measurements per patient. The cutoff of $25 \%$ corresponded to the median CV\% in our study cohort (25\%, IQR 18-35\%) and was comparable to cutoffs determined by receiver operating characteristic (ROC) curve analysis. High TacIPV was associated with higher risk of $d n \mathrm{DSA}$ development (HR 3.4, 95\% CI 1.0-11.1, $P=0.047$; Kaplan-Meier analysis $P=0.018$ ) and any kind of rejection episodes (HR 4.1, 95\% CI 1.1-14.8, $P=0.033$; Kaplan-Meier analysis $P=0.010)$. There was a clear trend towards higher TacIPV below the age of 6 years. TacIPV (CV\%) was stable over time. A TacIPV (CV\%) cutoff of 30\% or IPV quantification by mean absolute deviation (MAD) showed comparable results.

Conclusions High TacIPV is associated with an increased risk of $d n$ DSA development and rejection episodes $>$ year 1 posttransplant even in patients with low immunological risk profile. Therefore, in patients with high TacIPV, potential causes should be addressed, and if not resolved, changes in immunosuppressive therapy should be considered.
\end{abstract}

Keywords Tacrolimus intra-patient variability $($ TacIPV) $\cdot$ De novo donor-specific HLA antibody $(d n D S A) \cdot$ Allograft rejection $\cdot$ Kidney transplantation $(\mathrm{KTx}) \cdot$ Pediatrics

$\begin{array}{ll}\text { Abbreviations } \\ \text { ABMR } & \text { Antibody-mediated rejection } \\ \text { CNI } & \text { Calcineurin inhibitor } \\ \text { CV\% } & \text { Coefficient of variation in percent } \\ d n D S A & \text { De novo donor-specific antibodies }\end{array}$

Maral Baghai Arassi

maral.baghai@embl.de

1 Department of Pediatrics I, University Children's Hospital Heidelberg, Heidelberg, Germany

2 Structural and Computational Biology Unit, European Molecular Biology Laboratory, Heidelberg, Germany

3 Institute of Immunology, Transplantation Immunology, University Hospital Heidelberg, Heidelberg, Germany
eGFR Estimated glomerular filtration rate

HLA Human leukocyte antigen

KTx Kidney transplantation

MAD Mean absolute deviation

MDRD Modification of diet in renal disease

MFI Mean fluorescence intensity

MMF Mycophenolate mofetil

MPA Mycophenolic acid

Tac Tacrolimus

TacIPV Tacrolimus intra-patient variability

TCMR T cell-mediated rejection 


\section{Introduction}

Kidney transplantation (KTx) is the treatment of choice for pediatric patients with chronic kidney disease stage 5. The introduction of potent immunosuppressants such as the calcineurin inhibitor (CNI) tacrolimus (Tac) and the anti-metabolite mycophenolate mofetil (MMF) as well as anti-infective prophylaxis have substantially improved short-term transplant outcome. However, long-term graft survival remains limited [1]. While previously CNIinduced nephrotoxicity was considered the predominant reason for late graft function deterioration over time, growing evidence suggests that the causes are multifactorial and complex [2]. In this context, alloantigen-dependent factors, more specifically, de novo donor-specific antibodies ( $d n \mathrm{DSA})$ directed against human leukocyte antigens (HLA) play a major role [3]. Development of $d n \mathrm{DSA}$ is strongly associated with antibody-mediated rejection (ABMR) [4], one of the most common causes of late kidney allograft failure [5]. The exact mechanisms promoting $d n \mathrm{DSA}$ development are not yet fully understood; however, high Tac intra-patient variability (TacIPV) has recently been described as a potential contributing factor [6].

Tac is the cornerstone of modern immunosuppressive regimens. However, its narrow therapeutic index and high inter- and intraindividual variability represent a major challenge [7]. TacIPV describes fluctuations of a patient's Tac blood trough levels over time and is impacted by multiple factors including diet, diarrhea, genetics, drug-drug interactions, drug formulation, and non-adherence [8]. Although a causal link is still missing, it is generally assumed that variable Tac exposure results in periodic under- or overexposure contributing to immune activation and nephrotoxicity, both of which negatively impact graft function [8].

In the context of adult KTx, the association between TacIPV, poor graft survival, and alloimmunity has been widely established [9-13]. In the pediatric transplant population, however, this link is less well described. Few retrospective studies demonstrated a correlation between TacIPV and alloimmunity in pediatric kidney transplant patients, but they mainly focused on either non-Caucasians, living kidney donor recipients, or patients with high immunological risk profile [14-17]. Therefore, the impact of TacIPV in Caucasian pediatric kidney transplant patients with a low immunological risk profile remains poorly understood. Furthermore, this patient population is heterogenous with regard to age, body size, and biological and psychosocial maturation, all factors which could influence TacIPV. We therefore investigated in this retrospective cohort study the association between TacIPV, $d n \mathrm{DSA}$, rejection episodes, and graft function deterioration in pediatric kidney transplant recipients with a low immunological risk profile and analyzed whether TacIPV is associated with age.

\section{Patients and methods}

\section{Study design and patient population}

We conducted a retrospective observational single-center cohort study including 48 pediatric kidney transplant patients. All patients underwent kidney transplantation in the University Hospital Heidelberg, Germany, during 2006-2016. All included patients were considered as having a low immunological risk. Low immunological risk was defined as recipients of living-related donor kidney and/or at least 3 HLA matches of which at least 1 was an HLA-DR match. Patients with re-transplantation, multi-organ transplantation, positive crossmatch, less than 3 HLA matches, less than 1 HLA-DR match, and/or ABO-incompatible kidney transplantation were excluded. Other inclusion criteria were (i) HLA-DSA negativity pretransplant and within the first year posttransplant, (ii) absence of a biopsyproven rejection between month 6 and 12 posttransplant, (iii) at least three outpatient Tac trough level measurements between month 6 and 12 posttransplant, and (iv) a tacrolimus-based immunosuppressive regimen throughout the whole follow-up period. Patients were followed-up for up to 5 years posttransplant.

Standard immunosuppressive therapy at our study center consisted of a triple immunosuppressive regimen with Tac, MMF, and methylprednisolone; the majority of patients (77\%) did not receive induction therapy. Target immunosuppressive blood trough levels for Tac were slowly tapered from $10-12 \mu \mathrm{g} / \mathrm{L}$ in the first month to $5-8 \mu \mathrm{g} / \mathrm{L}$ after 6 months posttransplant. If given with everolimus $(n=4)$, target immunosuppressive blood trough levels for Tac were the following: $5-8 \mu \mathrm{g} / \mathrm{L}$ in the first month posttransplant, 4-7 $\mu \mathrm{g} / \mathrm{L}$ until month $4,3-5 \mu \mathrm{g} / \mathrm{L}$ thereafter. The initial MMF dose was $1200 \mathrm{mg} / \mathrm{m}^{2}$ body surface area per day, after 2 weeks posttransplant $600-900 \mathrm{mg} / \mathrm{m}^{2}$ per day, adjusted to achieve an MPA-AUC of $>40 \mathrm{mg} * \mathrm{~h} / \mathrm{L}$. Methylprednisolone therapy was tapered to $3 \mathrm{mg} / \mathrm{m}^{2}$ (max. $5 \mathrm{mg}$ /day) after 2 months posttransplant.

\section{Data collection}

Patient demographic and baseline transplantation characteristics were collected from medical charts. In addition, data on Tac whole-blood trough levels were collected from our electronic hospital laboratory information system. Only outpatient Tac levels measured between month 6 
and 12 posttransplant were included. In case of concentrations $<3 \mu \mathrm{g} / \mathrm{L}$ or $>20 \mu \mathrm{g} / \mathrm{L}$, patient charts were manually reviewed. Erroneously high trough levels resulting from drug intake before blood sampling were excluded. TacIPV was expressed as percentage and calculated as coefficient of variation $(\mathrm{CV} \%)$ based on the formula TacIPV $=\mathrm{CV} \%=$ (stand ard deviation $/$ mean $) \times 100$. Unless otherwise stated, TacIPV is used synonymously with Tac $\mathrm{CV} \%$ for the purpose of this study.

To compare TacIPV quantified by CV\% to other statistical measures of TacIPV, we additionally quantified the Tac mean absolute deviation (MAD) based on the formula MAD $\%=\left\{\left[\left(X_{\text {mean }}-X_{1}\right)+\left(X_{\text {mean }}-X_{2}\right) \ldots+\left(X_{\text {mean }}-X_{n}\right)\right] \div n\right\} \div$ $X_{\text {mean }}$, where $X$ is the Tac blood trough level.

Assessment of $d n \mathrm{DSA}$ was done at least yearly and during each episode of graft function deterioration and before initiation of anti-rejection therapy using LABScreen Luminex kits of One Lambda (Canoga Park, CA, USA), which use single HLA-antigen-coated beads and enable identification of immunoglobulin $\mathrm{G}(\mathrm{IgG})$ alloantibody specificities against HLA-A, -B, -C, -Cw, -DR, -DQ, and -DP antigens, as previously described [18-20]. A mean fluorescence intensity (MFI) cutoff value of $>500$ was considered positive. The cutoff of $500 \mathrm{MFI}$ is based on a Collaborative Transplant Study (CTS) analysis of HLA antibody cutoffs in patients with and without graft failure, which was performed in the Department of Transplantation Immunology Heidelberg. In this large multicenter study, the incidence of weak $d n$ DSA or non-DSA at a mean fluorescence intensity of 500 or higher was found to be higher in the graft loss than in the nonrejector group (76 vs. $40 \%, P<0.001$ ) [19]. In addition, kidney transplant function was assessed by the estimated glomerular filtration rate (eGFR) (modified Schwartz formula if $<18$ years or Modification of Diet in Renal Disease (MDRD) formula if $\geq 18$ years) at least every 3-6 months. Biopsy-proven rejection episodes were monitored throughout the entire follow-up period and graded using the Banff 2009-15 criteria [21]. Kidney biopsies were carried out as indication biopsies due to an increase of serum creatinine ( $>20 \%$ above baseline without an alternative explanation) and/or de novo persistent proteinuria $>100 \mathrm{~g}$ protein $/ \mathrm{mol}$ creatinine. No protocol biopsies were performed. No patient was treated for rejection without biopsy-proven diagnosis of rejection.

\section{Study outcome}

Primary study outcome was the association between TacIPV (months 6 to 12) and the development of $d n$ DSA $>$ year 1 posttransplant. Secondary outcome criteria were the association between TacIPV and (i) biopsy-proven rejection episodes, and (ii) graft function deterioration defined as $>50 \%$ decrease in eGFR compared to baseline (value at 4 weeks posttransplant) and/or eGFR $<30 \mathrm{~mL} / \mathrm{min}$ per $1.73 \mathrm{~m}^{2}$. In addition, we investigated the distribution of TacIPV across three different age groups $(2-5,6-11,12-21$ years).

\section{Statistical analysis}

Data are expressed as mean \pm SD unless otherwise stated. All analyses were performed using IBM SPSS Statistics version 27 (IBM, Corp, Armonk, NY). Differences between groups were assessed using either paired $t$-test, one-way analysis of variance, Kruskall-Wallis test, Mann-Whitney $U$-test, or chi-square test as appropriate. Bonferroni correction was used for post hoc analysis. A two-sided $P$ value $<0.05$ was considered statistically significant. Recipients were divided into two groups, one low TacIPV and one high TacIPV group, according to the CV\% cutoff of $25 \%$ corresponding to the median of TacIPV in the entire cohort. Kaplan-Meier survival analysis was performed to calculate the time to event from transplantation. For comparison of different groups, the log rank test was used. To examine risk factors for $d n$ DSA development, univariate and multivariable Cox regression analyses were performed. Factors included in the final multivariable model with forward selection were donor type (living or deceased donor), steroidfree therapy one year posttransplant, high TacIPV, and cold ischemia time. Receiver operating characteristic (ROC) plots for $\mathrm{CV} \%$ and MAD were generated, and area under the curve (AUC) and 95\% confidence interval (CI) limits were calculated using the method of Hanley and McNeil [22]. Optimum cutoff level for Tac CV\% was defined by the Youden Index. Biopsy-proven rejection episodes were analyzed as the pooled outcome criterion "any kind of rejection episode," including ABMR, T-cell-mediated rejection (TCMR), and borderline rejections, because the respective number of different rejection categories was too low for a meaningful analysis.

\section{Results}

\section{Clinical and demographic characteristics}

A total of 48 patients, 24 girls (50.0\%) and 24 boys (50.0\%), undergoing kidney transplantation between 2006 and 2016 were included. Median age at transplantation was 11.3 (5.6-15.7) years. All patients were of Caucasian descent. In the majority of patients, the primary kidney disease was congenital anomalies of the kidney and urinary tract (CAKUT, $47.9 \%$ ), followed by hereditary cystic diseases $(14.6 \%)$ and glomerular diseases (12.5\%). Twenty-one (43.8\%) patients received a kidney from a living donor. All patients received Tac, MMF, and methylprednisolone as initial immunosuppressive regimen. Eight patients $(16.7 \%)$ received additional 
induction therapy with basiliximab and 3 patients $(6.3 \%)$ were administered daclizumab. The average follow-up time was $57 \pm 5$ months posttransplant.

We collected a total of 566 outpatient Tac trough level values with a median of $11.0(6.0-17.0)$ measurements per patient. The mean Tac trough level was $6.6 \pm 1.2$, and the median TacIPV was 25\% (IQR 18-35\%). The cutoff value for high TacIPV was set at $25 \%$ corresponding to the median TacIPV in our study cohort. Baseline clinical and demographic characteristics for the high and low TacIPV cohort are shown in Table 1. There were no significant differences in baseline clinical and demographic characteristics (age, gender, HLA-matching, induction therapy, number of Tac trough levels, follow-up time) between the two cohorts. However, the low TacIPV cohort included significantly more living donor kidney transplant recipients $(P=0.018)$ and associated with that a significantly shorter cold ischemia time $(P=0.016)$.

\section{TacIPV and dnDSA}

Fifteen patients (31.3\%) developed $d n \mathrm{DSA}$ at a median of 37 (IQR 31-50) months posttransplant. Table 2 shows the characteristics of the $d n \mathrm{DSA}$ in the immunoglobulin $\mathrm{G}$ single-bead antibody (IgG-SAB) test. Supplementary Table 1 shows the characteristics of donor-specific HLA antibodies in the immunoglobulin $\mathrm{G}$ single-bead antibody (IgG-SAB) test stratified according to TacIPV status. The $d n \mathrm{DSA}$ positive cohort had a significantly longer cold ischemia time $(P=0.002)$ than the $d n$ DSA-negative cohort which was associated with the higher number of deceased kidney donor recipients in the $d n$ DSA-positive group. In addition, the $d n$ DSA-positive cohort had a significantly lower number of patients with steroid therapy at year 1 posttransplant $(P=0.013)$. Other baseline clinical and demographic characteristics between patients developing $d n$ DSA and those without did not differ significantly (Table 3 ).

Table 1 Baseline clinical and demographic characteristics of transplant patients stratified by a TacIPV cutoff value (CV\%) of $25 \%$

\begin{tabular}{|c|c|c|c|c|}
\hline Baseline characteristics & $\begin{array}{l}\text { Entire cohort } \\
N=48\end{array}$ & $\begin{array}{l}\text { Low TacIPV } \\
N=25\end{array}$ & $\begin{array}{l}\text { High TacIPV } \\
N=23\end{array}$ & $P$ value \\
\hline Cold ischemia time (hrs), mean $\pm \mathrm{SD}$ & $10.0 \pm 7.1$ & $7.6 \pm 5.8$ & $12.5 \pm 7.6$ & 0.016* \\
\hline Living donor, $n(\%)$ & $21(43.8)$ & $15(60.0)$ & $6(26.1)$ & 0.018* \\
\hline Pre-emptive donation, $n(\%)$ & $14(29.2)$ & $11(44.0)$ & $3(13.0)$ & 0.018* \\
\hline Recipient age, median (IQR) & $11.3(5.6-15.7)$ & $12.6(6.5-15.7)$ & $9.9(4.6-16.0)$ & 0.556 \\
\hline Female recipient, $n(\%)$ & $24(50.0)$ & $14(56.0)$ & $10(43.5)$ & 0.386 \\
\hline Primary kidney disease, $n(\%)$ & & & & 0.853 \\
\hline CAKUT & $23(47.9)$ & $13(52.0)$ & $10(43.5)$ & \\
\hline Glomerular disease & $6(12.5)$ & $3(12.0)$ & $3(13.0)$ & \\
\hline Cystic disease & $7(14.6)$ & $4(16.0)$ & $3(13.0)$ & \\
\hline Other & $12(25.0)$ & $5(20.0)$ & $7(30.4)$ & \\
\hline \multicolumn{5}{|l|}{ Number of HLA mismatches, mean \pm SD } \\
\hline $\mathrm{A} / \mathrm{B} / \mathrm{DR}$ & $2.02 \pm 1.02$ & $1.96 \pm 1.10$ & $2.09 \pm 0.95$ & 0.672 \\
\hline A & $0.58 \pm 0.58$ & $0.60 \pm 0.58$ & $0.57 \pm 0.59$ & 0.837 \\
\hline $\mathrm{B}$ & $0.85 \pm 0.55$ & $0.80 \pm 0.58$ & $0.91 \pm 0.52$ & 0.479 \\
\hline DR & $0.58 \pm 0.50$ & $0.56 \pm 0.51$ & $0.61 \pm 0.50$ & 0.739 \\
\hline Induction therapy, $n(\%)$ & $11(2.1)$ & $7(28.0)$ & $4(17.4)$ & 0.431 \\
\hline \multicolumn{5}{|l|}{ Immunosuppressive therapy at 1 year, $n(\%)$} \\
\hline Everolimus & $4(8.3)$ & $3(12.0)$ & $1(4.4)$ & 0.338 \\
\hline Azathioprine & $3(6.3)$ & $0(0)$ & $3(13.0)$ & 0.062 \\
\hline MMF & $39(81.3)$ & $22(88.0)$ & $17(73.9)$ & 0.212 \\
\hline Steroids & $43(89.6)$ & $24(96.0)$ & $19(82.6)$ & 0.129 \\
\hline Tacrolimus & $48(100)$ & $25(100)$ & $23(100)$ & \\
\hline $\begin{array}{l}\text { Number of tacrolimus trough levels month 6-12 posttransplant per } \\
\text { patient, median (IQR) }\end{array}$ & $11.0(6.0-17.0)$ & $10.0(5.5-17.0)$ & $11.0(7.0-17.0)$ & 0.501 \\
\hline Tacrolimus trough level 6-12 months posttransplant, mean \pm SD & $6.6 \pm 1.2$ & $6.5 \pm 1.2$ & $6.8 \pm 1.2$ & 0.336 \\
\hline Follow-up time (months), mean $\pm \mathrm{SD}$ & $57 \pm 5$ & $57 \pm 6$ & $57 \pm 5$ & 0.779 \\
\hline
\end{tabular}

TacIPV, tacrolimus intra-patient variability; $h r s$, hours; $n$, number of patients; $S D$, standard deviation; IQR, interquartile range; $C A K U T$, congenital anomalies of kidney and urinary tract; $H L A$, human leukocyte antigen, $* P<0.05$

Bold print and an asterix for $P$ values indicate a significant $P$ value defined as $P<0.05$ 
Table 2 Characteristics of donor-specific HLA antibodies in the immunoglobulin $\mathrm{G}$ single-bead antibody (IgG-SAB) test

\begin{tabular}{ll}
\hline Characteristics & $\begin{array}{l}d n \text { DSA-positive } \\
\text { cohort }(N=15)\end{array}$ \\
\hline Only class I, $n(\%)$ & $2(13.3)$ \\
Only class II, $n(\%)$ & $8(53.3)$ \\
Class I and II, $n(\%)$ & $5(33.3)$ \\
MFI class I (median, IQR) & $1486(769-2981)$ \\
MFI class II (median, IQR) & $2217(1262-9607)$ \\
Number DQ $\geq 1, n(\%)$ & $9(60)$ \\
Number DR $\geq 1, n(\%)$ & $5(33.3)$ \\
Number DP $\geq 1, n(\%)$ & $3(20.0)$ \\
Number A $\geq 1, n(\%)$ & $3(20.0)$ \\
Number B $\geq 1, n(\%)$ & $2(13.3)$ \\
Number Cw $\geq 1, n(\%)$ & $4(26.7)$ \\
Number C $\geq 1, n(\%)$ & $1(6.7)$ \\
Number of loci with at least one $d n$ DSA per & $1(1-3)$ \\
patient (median, IQR) & \\
\hline
\end{tabular}

dnDSA, de novo donor-specific HLA antibodies; class I, HLA class I; class II, HLA class II; Number, number of DSA directed against different HLA antigens; MFI, mean fluorescence intensity

Our cutoff value of $25 \%$ was comparable to the optimal TacIPV cutoff value determined by ROC curve analysis (Youden Index 26\%), for which an area under the ROC curve value (AUROC) of 0.727 (95\% CI 0.579-0.876; $P=0.012$ ) indicated a moderate capacity to discriminate between patients developing $d n \mathrm{DSA}$ and those without (Fig. 1). Quantifying TacIPV based on Tac MAD did not show any additional discriminatory benefit (AUROC 0.715; $95 \%$ CI $0.565-0.865 ; P=0.018)$.

Based on the TacIPV cutoff value of $25 \%$, Kaplan-Meier analysis revealed a strong association of high TacIPV with $d n \mathrm{DSA}$ development after the first year posttransplant $(P=0.018$, Fig. 2). Multivariable Cox regression analysis revealed a hazard ratio of 3.4 (95\% CI 1.0-11.1, $P=0.047$, Table 4) for $d n \mathrm{DSA}$ development. When we repeated this calculation with an alternative TacIPV cutoff value of $30 \%$, this association remained significant (Kaplan-Meier analysis $P=0.034$, multivariable Cox regression $P=0.043$ ).

\section{Association of TacIPV with rejection episodes and graft function deterioration}

Late rejection episodes beyond the first year posttransplant occurred in 13 transplant recipients $(27.1 \%)$. Median time to rejection episode was 37 months (IQR 27-50). Rejection types consisted of chronic active ABMR $(N=3(23.1 \%))$, acute TCMR $(N=2(15.4 \%))$, borderline rejection $(N=6$ (46.2)), and chronic TCMR $(N=2$ (15.4)). High TacIPV was significantly associated with biopsy-proven rejection episodes beyond the first year posttransplant (Kaplan-Meier analysis $P=0.010$, Fig. 2). Cox regression analysis revealed a hazard ratio of 4.1 (95\% CI 1.1-14.8, $P=0.033)$. Although a clear trend was visible, the association did not remain significant with an alternative TacIPV cutoff value of $30 \%$ (Kaplan-Meier analysis $P=0.058$, multivariable Cox regression $P=0.069)$. Our cutoff value of $25 \%$ was comparable to the optimal TacIPV cutoff value determined by ROC curve analysis (Youden Index 24\%) with an AUROC of 0.741 (95\% CI 0.593-0.888; $P=0.011$, Fig. 1). TacIPV quantification based on Tac MAD showed a comparable discriminatory power between patients developing any kind of rejection episodes and those who did not (AUROC $0.734 ; 95 \% \mathrm{CI}$ $0.577-0.891 ; P=0.013)$.

There was no significant association between TacIPV and graft function deterioration (Fig. 2). No graft losses or deaths occurred in this study population.

\section{TaclPV and patient age}

To address age heterogeneity of the pediatric patient population, we stratified our study cohort into 3 different age groups (2-5 years, 6-12 years, and $>12$ years). Although not statistically significant, we detected a clear trend towards a higher TacIPV with younger age, especially after removal of one extreme value in the oldest age group $(P=0.114$ before, $P=0.074$ after extreme value removal, Fig. 3).

\section{Stability of TaclPV over time posttransplant}

As a significant number of transplant-associated complications occurred $\geq 24$ months posttransplant, we evaluated whether a high TacIPV between months 6 and 12 posttransplant translated into high TacIPV at later timepoints. In fact, CV\% values in the time intervals 6-12 months (median 0.25), 13-18 months (median 0.26), and 19-24 months (median 0.28 ) posttransplant did not differ significantly $(p=0.831)$.

\section{Discussion}

In this study, we demonstrated that an increased TacIPV is significantly associated with the development of $d n \mathrm{DSA}$ and allograft rejection in a Caucasian pediatric kidney transplant cohort with low immunological risk profile. TacIPV can be quantified by several statistical measures, and standardization is still missing. Each approach has a different set of advantages and disadvantages; however, the Tac CV\% is one of the most widely used approaches, especially in the pediatric transplant population [14-16, 23-25]. In our study, we did not detect any difference in discriminatory power for development of $d n \mathrm{DSA}$ and allograft rejection between Tac CV\% and Tac MAD. However, large prospective studies 
Table 3 Baseline clinical and demographic characteristics of transplant patients stratified by $d n$ DSA development

\begin{tabular}{|c|c|c|c|}
\hline Baseline characteristics & $\begin{array}{l}d n \text { DSA positive } \\
N=15\end{array}$ & $\begin{array}{l}d n \text { DSA negative } \\
N=33\end{array}$ & $P$ value \\
\hline Cold ischemia time (hrs), mean $\pm \mathrm{SD}$ & $14.4 \pm 7.2$ & $7.9 \pm 6.1$ & $0.002 *$ \\
\hline Living donor, $n(\%)$ & $4(26.7)$ & $17(51.5)$ & 0.108 \\
\hline Pre-emptive transplantation, $n(\%)$ & $2(13.3)$ & $12(36.4)$ & 0.104 \\
\hline Recipient age, median (IQR) & $10.7(4.1-14.1)$ & $12.6(6.0-16.4)$ & 0.519 \\
\hline Female recipient, $n(\%)$ & $6(40.0)$ & $18(54.5)$ & 0.350 \\
\hline Primary kidney disease, $n(\%)$ & & & 0.211 \\
\hline CAKUT & $5(33.3)$ & $18(54.5)$ & \\
\hline Glomerular disease & $4(26.7)$ & $2(6.1)$ & \\
\hline Cystic disease & $2(13.3)$ & $5(15.2)$ & \\
\hline Other & $4(27.7)$ & $8(24.2)$ & \\
\hline \multicolumn{4}{|l|}{ Number of HLA mismatches, mean \pm SD } \\
\hline $\mathrm{A} / \mathrm{B} / \mathrm{DR}$ & $2.33 \pm 0.49$ & $1.88 \pm 1.17$ & 0.155 \\
\hline A & $0.80 \pm 0.68$ & $0.48 \pm 0.51$ & 0.079 \\
\hline $\mathrm{B}$ & $0.87 \pm 0.35$ & $0.85 \pm 0.62$ & 0.916 \\
\hline DR & $0.67 \pm 0.49$ & $0.55 \pm 0.51$ & 0.441 \\
\hline Induction therapy, $n(\%)$ & $4(26.7)$ & $7(21.1)$ & 0.665 \\
\hline \multicolumn{4}{|l|}{ Immunosuppression at year $1, n(\%)$} \\
\hline Everolimus & $0(0)$ & $4(12.1)$ & 0.159 \\
\hline Azathioprine & $2(13.3)$ & $1(3.0)$ & 0.172 \\
\hline MMF & $11(73.3)$ & $28(84.9)$ & 0.343 \\
\hline Steroids & $11(73.3)$ & $32(97.0)$ & 0.013* \\
\hline $\begin{array}{l}\text { Number of tacrolimus trough levels per patient in months } 6-12 \text { posttrans- } \\
\text { plant, median (IQR) }\end{array}$ & $13.0(8.0-18.0)$ & $9.0(6.0-16.5)$ & 0.208 \\
\hline Tacrolimus trough level in months $6-12$ posttransplant, mean \pm SD & $6.7 \pm 1.1$ & $6.6 \pm 1.3$ & 0.868 \\
\hline Follow-up time (months), mean $\pm \mathrm{SD}$ & $56 \pm 6$ & $57 \pm 5$ & 0.474 \\
\hline
\end{tabular}

$d n D S A$, de novo donor-specific antibodies; $n$, number of patients; $h r s$, hours; $S D$, standard deviation; IQR, interquartile range; $C A K U T$, congenital anomalies of kidney and urinary tract; $H L A$, human leukocyte antigen, $* P<0.05$

Fig. 1 ROC curve analysis to determine the optimal TacIPV cutoff value differentiating between patients with increased alloimmune response defined as either $d n$ DSA development (a) or biopsy-proven rejection episodes (b) and those without. TacIPV is quantified by coefficient of variation $(\mathrm{CV} \%$, red line) and mean absolute deviation (MAD, blue line) a dnDSA development

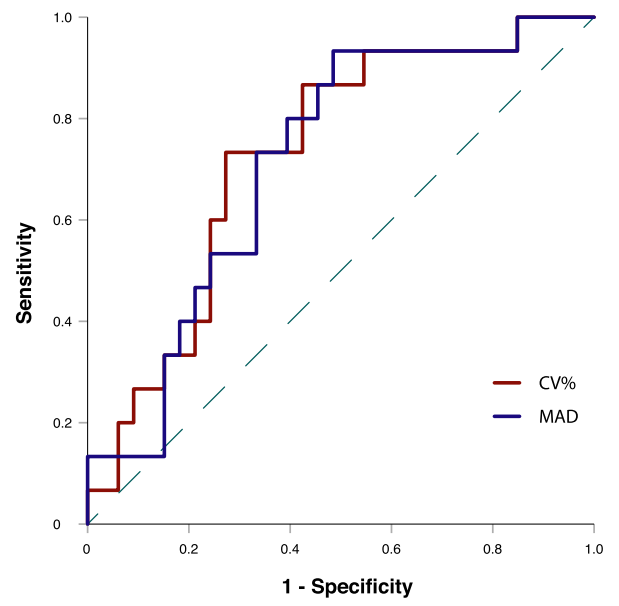

b

Rejection episodes

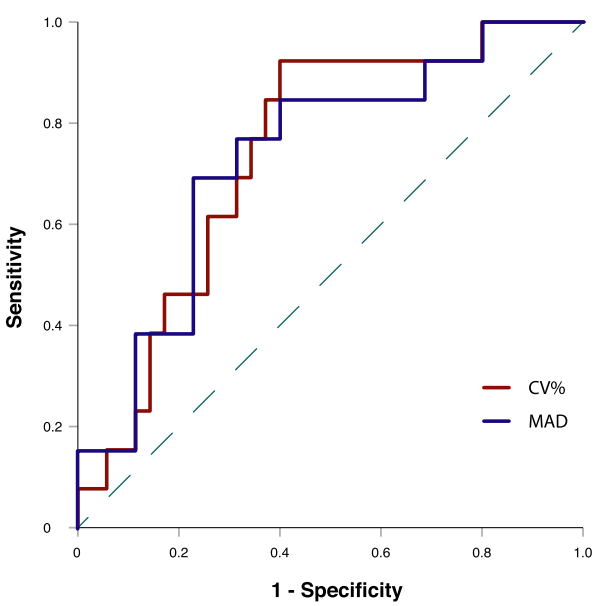


Fig. 2 Association of tacrolimus intra-patient variability (TacIPV) with de novo donorspecific HLA antibody development ( $d n \mathrm{DSA})(\mathbf{a})$, biopsyproven rejection episodes (b), and graft function deterioration (c) defined as $>50 \%$ decrease in eGFR compared to baseline and/or eGFR $<30 \mathrm{~mL} / \mathrm{min}$ per $1.73 \mathrm{~m}^{2}$ beyond the first year posttransplant. Log rank $P$ values of Kaplan-Meier analysis are shown a

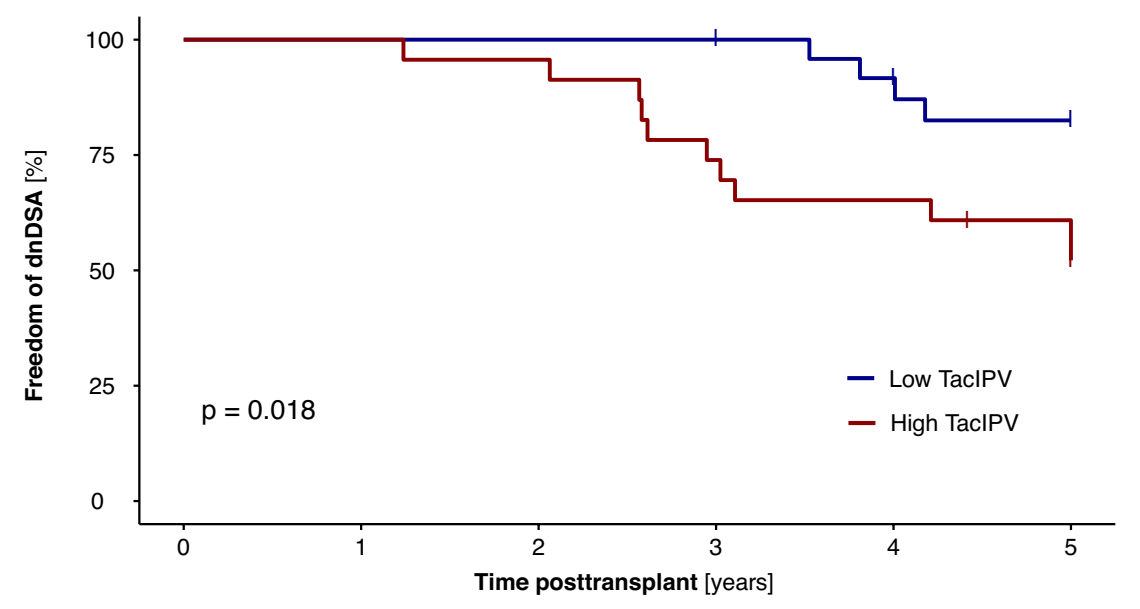

No. at risk

$\begin{array}{rrrrrr}\text { Low TaclPV } & 25 & 25 & 25 & 22 & 18 \\ \text { High TaclPV } & 23 & 22 & 17 & 15 & 13\end{array}$

b

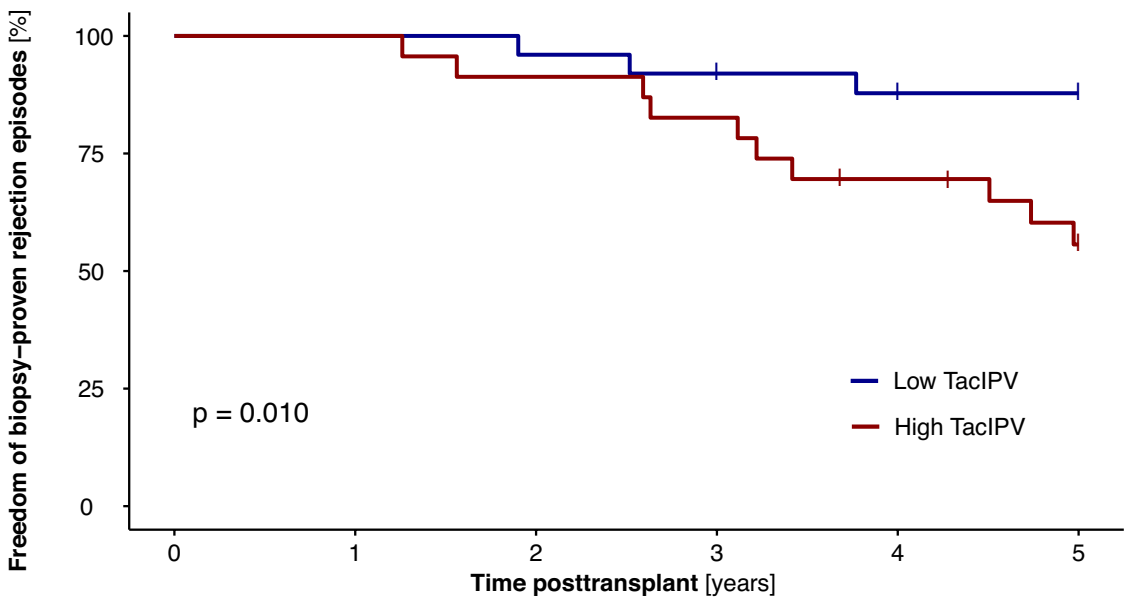

No. at risk

\begin{tabular}{|c|c|c|c|c|c|}
\hline Low TaclPV & 25 & 24 & 23 & 21 & 19 \\
\hline High TacIPV & 23 & 21 & 19 & 15 & \\
\hline
\end{tabular}

\section{C}

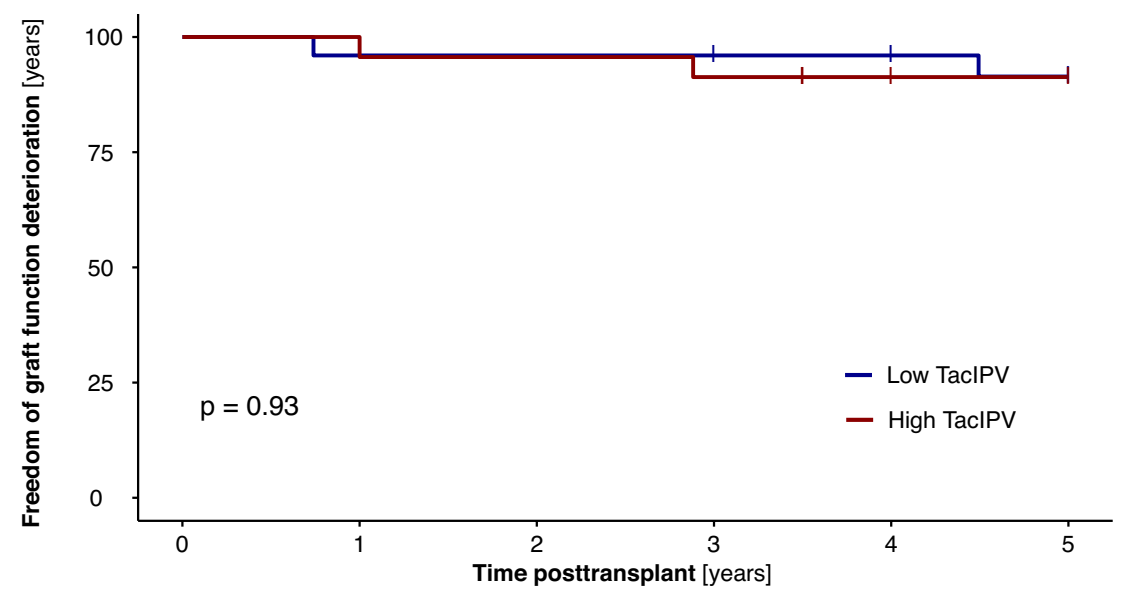

No. at $r$

Low TacIPV

High TaclPV

24

24

24

23

20

23
21

19 
Table 4 Risk factor analysis for de novo donor-specific HLA antibody ( $d n$ DSA) development

\begin{tabular}{|c|c|c|c|c|}
\hline Risk factor & Unadjusted HR (95\% CI) & $P$ value & Adjusted HR (95\% CI) & $P$ value \\
\hline High TacIPV (\%) & $3.6(1.2-11.4)$ & $0.028 *$ & $3.4(1.0-11.1)$ & $0.047 *$ \\
\hline HLA-DR mismatches & & $0.394 *$ & & \\
\hline Cold ischemia time (hrs) $)^{\#}$ & $5.1(1.1-25.0)$ & $0.042 *$ & $3.9(1.1-14.2)$ & 0.038* \\
\hline Steroid-free therapy month 12 posttransplant & $5.9(1.9-18.8)$ & $0.003 *$ & $8.5(2.3-31.6)$ & 0.001* \\
\hline Donor type (living) & $0.4(0.1-1.1)$ & 0.08 & & \\
\hline Recipient age (years) & $1.0(0.9-1.1)$ & 0.702 & & \\
\hline Induction therapy & $1.6(0.5-5.2)$ & 0.416 & & \\
\hline Mean Tac trough concentration $(\mu \mathrm{g} / \mathrm{L})$ & $1.0(0.7-1.6)$ & 0.881 & & \\
\hline
\end{tabular}

$H R$, hazard ratio; $C I$, confidence interval; TacIPV, tacrolimus intra-patient variability quantified as Tac coefficient of variation (CV\%); $H L A$, human leukocyte antigen; * $P<0.05$; ${ }^{*} \log$ transformed because nonparametric with right screw

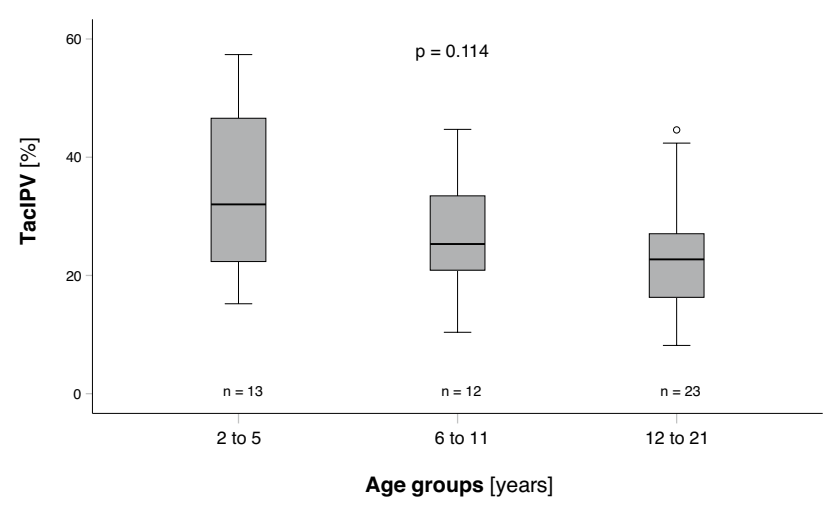

Fig. 3 Distribution of tacrolimus intra-patient variability (TacIPV) measured as coefficient of variation (CV\%) across three different age groups $(2-5,6-11,12-21$ years)

systematically assessing both quantification methods are needed to allow a thorough comparison of both methods.

There is no benchmark yet regarding which time period is best for TacIPV calculation. As the early phase posttransplant is characterized by high fluctuations in Tac blood levels and knowledge on the significance of TacIPV beyond 12 months after transplantation is limited, we focused on the time period between 6 and 12 months posttransplant [8, 24]. However, we could demonstrate that TacIPV is stable over time posttransplant, as high $\mathrm{CV} \%$ between months 6 and 12 posttransplant translated into high $\mathrm{CV} \%$ during the later posttransplant period. To our knowledge, this is the first study, in children and adults alike, showing that TacIPV is stable over time posttransplant, supporting the notion that TacIPV quantified between months 6 and 12 posttransplant could be used to predict transplant-associated events happening at later timepoints posttransplant. In fact, current literature indicates that $d n$ DSA development, likely resulting from inadequate immunosuppression, is responsible for a more indolent and slowly progressive form of allograft injury, in which chronic histological changes can take long periods of time before allograft function is visibly impaired [4].

Defining a clear cutoff value to determine high TacIPV is also currently debated. Table 5 offers an overview of reported TacIPV cutoffs determined by Tac CV\% in pediatric and adult kidney transplant studies. Most studies suggested a TacIPV cutoff of $30 \%$ for dividing patients into a high and low TacIPV cohort. Cutoffs were chosen empirically either based on the distribution of TacIPV values (i.e., quartiles, tertiles, or median) or based on ROC curve analyses. As in our study population, the median TacIPV was $25 \%$ and ROC curve analyses detected a similar optimal cutoff, we set the cutoff for high TacIPV at 25\%. As this cutoff is relatively low compared to other studies, we additionally investigated a higher cutoff of $30 \%$, which did not change the statistically significant association of TacIPV with $d n$ DSA development. However, most likely due to our low sample size and event number, we could only detect a strong trend towards an association of TacIPV with rejection episode development when choosing a higher CV\% of 30 .

The association between high TacIPV and graft outcome has been demonstrated in multiple studies. Borra et al. were among the first to show that high TacIPV is associated with worse kidney graft survival [9]. These findings were confirmed by several follow-up studies in adult patient cohorts revealing that high TacIPV is correlated with graft survival, rejection episodes, and $d n$ DSA formation [10-13, $27,28,32]$. In the pediatric patient cohort, Pollock-BarZiv et al. were the first to describe an association between high TacIPV and increased risk of late rejection and graft loss [17]. These findings were later validated in several other studies demonstrating a similar association of high TacIPV with late acute rejection [23,33] and graft loss [34]. Notably, Solomon et al. linked TacIPV to $d n$ DSA development in a minority cohort of mainly African American and Hispanic patients with low socioeconomic background [14]. A study by Aksoy et al. supported these findings and reported an association between high TacIPV and $d n$ DSA development 
Table 5 Reported TacIPV cutoffs measured by coefficient of variation (CV\%) starting at least $\geq 3$ months posttransplant in adult and pediatric kidney transplant studies

\begin{tabular}{|c|c|c|c|c|c|}
\hline Reference & TacIPV assessment period & TacIPV cutoff & Outcome parameter & Study population & $N$ \\
\hline Süsal et al., 2019 [26] & 1,2 , and 3 years posttransplant & $30 \%$ & Graft and patient survival & Adult & 6638 \\
\hline O’Regan et al., 2016 [27] & 3-12 months posttransplant & $\begin{array}{l}\text { Quartiles (median) } \\
\text { Q1: } 13 \% \\
\text { Q2: } 18 \% \\
\text { Q3: } 25 \% \\
\text { Q4: } 37 \%\end{array}$ & Graft and patient survival & Adult & 394 \\
\hline Rodrigo et al., 2016 [11] & 4-12 months posttransplant & $30 \%$ & $\begin{array}{l}\text { Graft survival and development } \\
\text { of } d n \mathrm{DSA}\end{array}$ & Adult & 310 \\
\hline Goodall et al., 2017 [28] & 6-12 months posttransplant & $\begin{array}{l}\text { Quartiles (cutoffs) } \\
\text { Q1: }<13 \% \\
\text { Q2: } \geq 13-18 \% \\
\text { Q3: } \geq 18-25 \% \\
\text { Q4: }>25 \%\end{array}$ & $\begin{array}{l}\text { Graft and patient survival and } \\
\text { rejection events }\end{array}$ & Adult & 628 \\
\hline Vanhove et al., 2016 [13] & 6-12 months posttransplant & $\begin{array}{l}\text { Tertile (mean) } \\
\text { T1: } 10 \% \\
\text { T2: } 18 \% \\
\text { T3: } 31 \%\end{array}$ & $\begin{array}{l}\text { Acute and chronic histologic } \\
\text { lesions }\end{array}$ & Adult & 220 \\
\hline Huang et al., 2016 [29] & $\leq 6$ months pre-biopsy & $18 \%$ & Rejection events & Adult & 161 \\
\hline Mo et al., 2019 [30] & $6-12$ months posttransplant & $21 \%$ & $\begin{array}{l}\text { Graft loss and chronic histologic } \\
\text { lesions }\end{array}$ & Adult & 671 \\
\hline Solomon et al., 2019 [14] & $\geq 3$ months posttransplant & $30 \%$ & $\begin{array}{l}\text { Development of } d n \text { DSA and } \\
\text { rejection events }\end{array}$ & Pediatric & 38 \\
\hline Pizzo et al., 2016 [15] & $\begin{array}{l}\geq 6 \text { months posttransplant } \\
\text { and } \leq 6 \text { months pre-biopsy }\end{array}$ & $31 \%$ & $\begin{array}{l}\text { Development of } d n \text { DSA and } \\
\text { rejection events }\end{array}$ & Pediatric & 23 \\
\hline Hsiau et al., 2011 [31] & $1-12$ months posttransplant & $41 \%$ & Rejection events & Pediatric & 46 \\
\hline Kaya Aksoy et al., 2019 [16] & $\begin{array}{l}\text { Period 1: }<6 \text { months posttrans- } \\
\text { plant } \\
\text { Period 2: 6-12 months post- } \\
\text { transplant } \\
\text { Period 3: } 1 \text { year-end of follow- } \\
\text { up }\end{array}$ & $32 \%$ & $\begin{array}{l}\text { Development of } d n \mathrm{DSA} \text { and } \\
\text { graft function }\end{array}$ & Pediatric & 67 \\
\hline
\end{tabular}

$d n \mathrm{DSA}$, de novo donor-specific antibodies; $n$, number of patients

in living kidney donor KTx [16]. To our knowledge, our study is the first to validate these findings in a cohort of lowrisk Caucasian patients including both living and deceased donor kidney transplantations. Similar to previous studies in the pediatric kidney transplant population [14-17], we detected a significant association between TacIPV and biopsy-proven rejection, underscoring the importance of TacIPV even in patients with low immunological risk profile. This association might be especially relevant for patients with relatively low Tac blood target levels as shown in our study population, where a low median Tac level combined with high TacIPV led to a considerable number of rejection episodes and $d n$ DSA development even in patients with low immunological risk.

We could not detect a significant association with graft function deterioration, probably due to the overall low incidence of this event and the relatively low number of patients.

Interestingly, we saw a clear trend towards a higher TacIPV in patients below the age of 6 years. This finding is in contrast to studies suggesting a higher TacIPV in adolescents due to lower therapy adherence $[31,35,36]$, but is consistent with a study by Prytula et al. [33]. Although our study does not allow any causal inferences, a possible explanation might be a generally higher susceptibility to infections, in particular gastrointestinal, at younger age, which is known to interfere with TacIPV [8] because diarrhea leads to increased Tac bioavailability. Other potential mechanisms are differences in the metabolism of Tac among pediatric age groups [37], the number of Tac dose changes, which has previously been associated in adult kidney transplant recipients with increased IPV $[9,38]$, age-specific differences in the frequency of infections and infection-associated inflammation [39], and/or age-specific differences in the composition of the gut microbiota which appears to contribute to Tac metabolism [40]. Future studies should therefore consider age-group stratification and detailed adherence and infection monitoring for an adequate assessment of the relationship between age and TacIPV. 
Other factors besides variability in Tac exposure are likely to have contributed to the development of $d n D \mathrm{SA}$ formation. In our patient cohort, the $d n \mathrm{DSA}$-positive subgroup had a longer cold ischemia time than the $d n \mathrm{DSA}-$ negative subgroup and less frequently received steroids at year 1 posttransplant than the $d n \mathrm{DSA}$-negative subgroup. However, in a large prospective randomized trial on steroid avoidance in pediatric kidney transplantation, steroid-free immunosuppression was not associated with a higher frequency of $d n$ DSA development [41].

Our study has several limitations. First, the investigated patient cohort is relatively small and findings may not be generalizable to highly heterogenous pediatric patient populations. Second, given the retrospective design of this study, we cannot make any statement about the causal link between TacIPV and $d n$ DSA formation as information on factors known to be associated with TacIPV such as non-adherence or infectious episodes was not available. Despite these limitations, our study provides important insights into the relationship between Tac variability, $d n \mathrm{DSA}$ development, and allograft rejection in a pediatric cohort with low immunological risk profile.

In conclusion, our data indicate that high TacIPV between months 6 and 12 posttransplant is, irrespective of quantification by $\mathrm{CV} \%$ or MAD, associated with a higher risk of developing $d n \mathrm{DSA}$ directed against HLA and allograft rejection beyond the first year posttransplant. However, further validation in large-scale, prospective trials is needed to qualify TacIPV as a predictive diagnostic marker for increased risk of alloimmunity. If accomplished, TacIPV could serve as a cheap and non-invasive biomarker for identifying patients at risk of developing $d n \mathrm{DSA}$ and long-term graft loss. For now, in patients with highly variable Tac trough blood concentrations, potential causes of high TacIPV should be addressed and if not resolved, a change in immunosuppressive therapy should be considered to prevent $d n \mathrm{DSA}$ development with the overall goal of preventing rejection and improving longterm graft outcomes.

Supplementary Information The online version contains supplementary material available at https://doi.org/10.1007/s00467-022-05426-3.

Acknowledgements We acknowledge funding from the Physician Scientist Program of the Medical Faculty of Heidelberg (to Maral Baghai Arassi).

Funding Open Access funding enabled and organized by Projekt DEAL.

\section{Declarations}

Ethics approval The study was approved by the Ethics Committee of the Medical Faculty of Heidelberg University and performed in accord- ance with the World Medical Association Declaration of Helsinki Ethical Principles in the currently valid version.

Conflict of interest The authors declare no competing interests.

Open Access This article is licensed under a Creative Commons Attribution 4.0 International License, which permits use, sharing, adaptation, distribution and reproduction in any medium or format, as long as you give appropriate credit to the original author(s) and the source, provide a link to the Creative Commons licence, and indicate if changes were made. The images or other third party material in this article are included in the article's Creative Commons licence, unless indicated otherwise in a credit line to the material. If material is not included in the article's Creative Commons licence and your intended use is not permitted by statutory regulation or exceeds the permitted use, you will need to obtain permission directly from the copyright holder. To view a copy of this licence, visit http://creativecommons.org/licenses/by/4.0/.

\section{References}

1. Gondos A, Döhler B, Brenner H, Opelz G (2013) Kidney graft survival in Europe and the United States: strikingly different longterm outcomes. Transplantation 95:267-274. https://doi.org/10. 1097/TP.0b013e3182708ea8

2. Neuberger JM, Bechstein WO, Kuypers DRJ, Burra P et al (2017) Practical recommendations for long-term management of modifiable risks in kidney and liver transplant recipients: a guidance report and clinical checklist by the Consensus on Managing Modifiable Risk in Transplantation (COMMIT) group. Transplantation 101:S1-S56. https://doi.org/10.1097/TP.0000000000001651

3. Wiebe C, Gibson IW, Blydt-Hansen TD, Karpinski M et al (2012) Evolution and clinical pathologic correlations of de novo donorspecific HLA antibody post kidney transplant: clinical pathologic correlations of de novo DSA. Am J Transplant 12:1157-1167. https://doi.org/10.1111/j.1600-6143.2012.04013.x

4. Loupy A, Hill GS, Jordan SC (2012) The impact of donor-specific anti-HLA antibodies on late kidney allograft failure. Nat Rev Nephrol 8:348-357. https://doi.org/10.1038/nrneph.2012.81

5. Sellarés J, de Freitas DG, Mengel M, Reeve J et al (2012) Understanding the causes of kidney transplant failure: the dominant role of antibody-mediated rejection and nonadherence. Am J Transplant 12:388-399. https://doi.org/10.1111/j.1600-6143.2011. 03840.x

6. Mendoza Rojas A, Hesselink DA, van Besouw NM, Baan C et al (2019) Impact of low tacrolimus exposure and high tacrolimus intra-patient variability on the development of de novo anti-HLA donor-specific antibodies in kidney transplant recipients. Expert Rev Clin Immunol 15:1323-1331. https://doi.org/10.1080/17446 66X.2020.1693263

7. Shuker N, van Gelder T, Hesselink DA (2015) Intra-patient variability in tacrolimus exposure: causes, consequences for clinical management. Transplant Rev 29:78-84. https://doi.org/10.1016/j. trre.2015.01.002

8. Gonzales HM, McGillicuddy JW, Rohan V, Chandler J et al (2020) A comprehensive review of the impact of tacrolimus intrapatient variability on clinical outcomes in kidney transplantation. Am J Transplant 20:1969-1983. https://doi.org/10.1111/ajt.16002

9. Borra LCP, Roodnat JI, Kal JA, Mathot R et al (2010) High within-patient variability in the clearance of tacrolimus is a risk factor for poor long-term outcome after kidney transplantation. Nephrol Dial Transplant 25:2757-2763. https://doi.org/10.1093/ ndt/gfq096 
10. Sapir-Pichhadze R, Wang Y, Famure O, Li Y et al (2014) Timedependent variability in tacrolimus trough blood levels is a risk factor for late kidney transplant failure. Kidney Int 85:1404-1411. https://doi.org/10.1038/ki.2013.465

11. Rodrigo E, Segundo DS, Fernández-Fresnedo G, López-Hoyos M et al (2016) Within-patient variability in tacrolimus blood levels predicts kidney graft loss and donor-specific antibody development. Transplantation 100:2479-2485. https://doi.org/10.1097/ TP.0000000000001040

12. Sablik KA, Clahsen-van Groningen MC, Hesselink DA, van Gelder T et al (2018) Tacrolimus intra-patient variability is not associated with chronic active antibody mediated rejection. PLoS One 13:e0196552. https://doi.org/10.1371/journal.pone.0196552

13. Vanhove T, Vermeulen T, Annaert P, Lerut E et al (2016) High intrapatient variability of tacrolimus concentrations predicts accelerated progression of chronic histologic lesions in renal recipients. Am J Transplant 16:2954-2963. https://doi.org/10.1111/ajt.13803

14. Solomon S, Colovai A, Del Rio M, Hayde N (2020) Tacrolimus variability is associated with de novo donor-specific antibody development in pediatric renal transplant recipients. Pediatr Nephrol 35:261-270. https://doi.org/10.1007/s00467-019-04377-6

15. Pizzo HP, Ettenger RB, Gjertson DW, Reed E et al (2016) Sirolimus and tacrolimus coefficient of variation is associated with rejection, donor-specific antibodies, and nonadherence. Pediatr Nephrol 31:2345-2352. https://doi.org/10.1007/ s00467-016-3422-5

16. Kaya Aksoy G, Comak E, Koyun M, Akbaş H et al (2019) Tacrolimus variability: a cause of donor-specific anti-HLA antibody formation in children. Eur J Drug Metab Pharmacokinet 44:539-548. https://doi.org/10.1007/s13318-019-00544-0

17. Pollock-Barziv SM, Finkelstein Y, Manlhiot C, Dipchand A et al (2010) Variability in tacrolimus blood levels increases the risk of late rejection and graft loss after solid organ transplantation in older children. Pediatr Transplant 14:968-975. https://doi.org/10. 1111/j.1399-3046.2010.01409.x

18. Fichtner A, Süsal C, Höcker B, Rieger S et al (2021) Association of non-HLA antibodies against endothelial targets and donorspecific HLA antibodies with antibody-mediated rejection and graft function in pediatric kidney transplant recipients. Pediatr Nephrol. https://doi.org/10.1007/s00467-021-04969-1

19. Süsal C, Wettstein D, Döhler B, Morath M et al (2015) Association of kidney graft loss with de novo produced donor-specific and non-donor-specific HLA antibodies detected by single antigen testing. Transplantation 99:1976-1980. https://doi.org/10.1097/ TP.0000000000000672

20. Fichtner A, Süsal C, Höcker B, Rieger S et al (2016) Association of C1q-fixing DSA with late graft failure in pediatric renal transplant recipients. Pediatr Nephrol 31:1157-1166. https://doi.org/ 10.1007/s00467-016-3322-8

21. Sis B, Mengel M, Haas M, Colvin RB et al (2010) Banff '09 meeting report: antibody mediated graft deterioration and implementation of Banff working groups. Am J Transplant 10:464-471. https://doi.org/10.1111/j.1600-6143.2009.02987.x

22. Hanley JA, McNeil BJ (1983) A method of comparing the areas under receiver operating characteristic curves derived from the same cases. Radiology 148:839-843. https://doi.org/10.1148/ radiology.148.3.6878708

23. Abu Bakar K, Mohamad NA, Hodi Z, McCulloch T et al (2019) Defining a threshold for tacrolimus intra-patient variability associated with late acute cellular rejection in paediatric kidney transplant recipients. Pediatr Nephrol 34:2557-2562. https://doi.org/ 10.1007/s00467-019-04346-Z

24. Kuypers DRJ (2020) Intrapatient variability of tacrolimus exposure in solid organ transplantation: a novel marker for clinical outcome. Clin Pharmacol Ther 107:347-358. https://doi.org/10. 1002/cpt.1618

25. Schumacher L, Leino AD, Park JM (2021) Tacrolimus intrapatient variability in solid organ transplantation: a multiorgan perspective. Pharmacother J Hum Pharmacol Drug Ther 41:103-118. https://doi.org/10.1002/phar.2480

26. Süsal C, Döhler B (2019) Late intra-patient tacrolimus trough level variability as a major problem in kidney transplantation: a collaborative transplant study report. Am J Transplant 19:28052813. https://doi.org/10.1111/ajt.15346

27. O'Regan JA, Canney M, Connaughton DM, O'Kelly P et al (2016) Tacrolimus trough-level variability predicts long-term allograft survival following kidney transplantation. J Nephrol 29:269-276. https://doi.org/10.1007/s40620-015-0230-0

28. Goodall DL, Willicombe M, McLean AG, Taube D (2017) High intrapatient variability of tacrolimus levels and outpatient clinic nonattendance are associated with inferior outcomes in renal transplant patients. Transplant Direct 3:e192. https://doi.org/10. 1097/TXD.0000000000000710

29. Huang C-T, Shu K-H, Ho H-C, Wu M-J (2016) Higher variability of tacrolimus trough level increases risk of acute rejection in kidney transplant recipients. Transplant Proc 48:1978-1980. https:// doi.org/10.1016/j.transproceed.2016.02.081

30. Mo H, Kim S-Y, Min S, Han A et al (2019) Association of intrapatient variability of tacrolimus concentration with early deterioration of chronic histologic lesions in kidney transplantation. Transplant Direct 5:e455. https://doi.org/10.1097/TXD.00000 00000000899

31. Hsiau M, Fernandez HE, Gjertson D, Ettenger R et al (2011) Monitoring nonadherence and acute rejection with variation in blood immunosuppressant levels in pediatric renal transplantation. Transplantation 92:918-922. https://doi.org/10.1097/TP. 0b013e31822dc34f

32. Rahamimov R, Tifti-Orbach H, Zingerman B, Green $\mathrm{H}$ et al (2019) Reduction of exposure to tacrolimus trough level variability is associated with better graft survival after kidney transplantation. Eur J Clin Pharmacol 75:951-958. https://doi.org/10.1007/ s00228-019-02643-y

33. Prytula AA, Bouts AH, Mathot RAA, van Gleder T et al (2012) Intra-patient variability in tacrolimus trough concentrations and renal function decline in pediatric renal transplant recipients. Pediatr Transplant 16:613-618. https://doi.org/10.1111/j.13993046.2012.01727.x

34. Gold A, Tönshoff B, Döhler B, Süsal C (2020) Association of graft survival with tacrolimus exposure and late intra-patient tacrolimus variability in pediatric and young adult renal transplant recipients-an international CTS registry analysis. Transpl Int 33:1681-1692. https://doi.org/10.1111/tri.13726

35. Rianthavorn P, Ettenger RB (2005) Medication non-adherence in the adolescent renal transplant recipient: a clinician's viewpoint. Pediatr Transplant 9:398-407. https://doi.org/10.1111/j.13993046.2005.00358.x

36. Feddersen N, Pape L, Beneke J, Brand K et al (2021) Adherence in pediatric renal recipients and its effect on graft outcome, a single-center, retrospective study. Pediatr Transplant 25:e13922. https://doi.org/10.1111/petr.13922

37. Prytuła A, van Gelder T (2019) Clinical aspects of tacrolimus use in paediatric renal transplant recipients. Pediatr Nephrol 34:3143. https://doi.org/10.1007/s00467-018-3892-8

38. Rozen-Zvi B, Schneider S, Lichtenberg S, Green H et al (2017) Association of the combination of time-weighted variability of tacrolimus blood level and exposure to low drug levels with graft survival after kidney transplantation. Nephrol Dial Transplant 32:393-399. https://doi.org/10.1093/ndt/gfw394 
39. Bonneville E, Gautier-Veyret E, Ihl C, Hilleret M et al (2020) Unexpected overdose blood concentration of tacrolimus: keep in mind the role of inflammation. Br J Clin Pharmacol 86:18881891. https://doi.org/10.1111/bcp.14292

40. Zimmermann M, Zimmermann-Kogadeeva M, Wegmann R, Goodman AL (2019) Mapping human microbiome drug metabolism by gut bacteria and their genes. Nature 570:462-467. https:// doi.org/10.1038/s41586-019-1291-3
41. Chaudhuri A, Goddard EA, Green M, Ardura MI (2020) Diarrhea in the pediatric solid organ transplantation recipient: a multidisciplinary approach to diagnosis and management. Pediatr Transplant 25:e13886. https://doi.org/10.1111/petr.13886

Publisher's note Springer Nature remains neutral with regard to jurisdictional claims in published maps and institutional affiliations. 